\title{
Rentenversicherung oder Kapitalmarkt? \\ Lebensstandardsicherung als Aufgabe einer zukunftsfähigen Alterssicherungspolitik
}

\author{
Gerhard Bäcker
}

\section{Alterssicherung - mehr als Armutsvermeidung}

Über Monate hinweg (letztlich über Jahre hinweg) ist die rentenpolitische Diskussion durch den Komplex „Grundrente “ geprägt worden. ${ }^{1}$ Noch sind viele Fragen offen. Vor allem bleibt abzuwarten, wie die Einkommensanrechnung, die die Rentenversicherung in Zusammenarbeit mit den Finanzbehörden vornehmen soll, geregelt werden kann und wie die Gegenfinanzierung aussehen soll ${ }^{2}$. Es ist jedoch davon auszugehen, dass bei der Rentenberechnung unter bestimmten Bedingungen (Versicherungsjahre, Mindest- und Maximalschwelle der Aufstockung, Kürzungsprozentsatz, Einkommensanrechnung auch in Paar-Haushalten) eine Aufwertung von niedrigen Entgeltpunkten für langjährig Versicherte durchgeführt wird, so dass niedrige Renten aufgestockt werden und damit näher an das Niveau der Grundsicherung im Alter heranreichen bzw. dieses auch übersteigen. Im Zusammenwirken mit der Einführung von Freibeträgen bei der Grundsicherung nicht nur wie bislang für Renten aus einer zusätzlichen, sondern additiv auch aus der verpflichtenden Altersvorsorge in der öffentlichen Rentenversicherung wird es zudem dazu kommen, dass viele Ältere (auch im Rentenbestand!) eine deutliche Verbesserung ihrer Einkommenslage erreichen. ${ }^{3}$ Hinzu kommen sollen Freibeträge beim Wohngeld. Eine

1 Über Jahre hinweg, da schon in den zurückliegenden Legislaturperioden verschiedene Ansätze einer Grundrente diskutiert wurden, die aber allesamt nicht umgesetzt werden konnten.

2 Vgl. dazu das Gutachten des Sozialbeirats zum Rentenversicherungsbericht 2019 (Sozialbeirat 2019).

3 Durch diese Form einer vorleistungsabhängigen Berechnung eines Grundsicherungsanspruchs zählen zukünftig auch ein Großteil jener Rentner*innen, die auf Grund der bislang vollen Einkommensanrechnung keine Grundsicherung bezogen haben, zum Kreis der Leistungsberechtigten und -empfänger. Allerdings nur 
genaue Bestimmung des begünstigten Personenkreises und des zu erwartenden Gesamteinkommens wird man allerdings erst vornehmen können, wenn ein Gesetzentwurf vorliegt. ${ }^{4}$

Wie auch immer die konkreten gesetzlichen Regelungen aussehen werden, sie werden Rückwirkungen haben auf ein zweites rentenpolitisches Diskussionsfeld, das mit den Stichworten „Lebensstandardsicherung“ und „Rentenniveau“ umschrieben werden kann. Im Frühjahr 2020 wird hier die von der Bundesregierung eingesetzte Rentenkommission „Verlässlicher Generationenvertrag" ihren Bericht vorlegen. Entsprechend dem Auftrag der Kommission geht es um Fragen der langfristigen Finanzierbarkeit und Ausgestaltung der Alterssicherung allgemein und der Rentenversicherung im Besonderen. Es werden Aussagen zu erwarten sein, welche Bedeutung dem Ziel der Lebensstandardsicherung zukommen soll, welche Entwicklung das Leistungsniveau der Rentenversicherung nimmt und wie die Rollenverteilung zwischen der Rentenversicherung einerseits und den kapitalbasierten Systemen andererseits aussehen soll.

Diese Fragen stehen im Mittelpunkt der folgenden Ausführungen. Wie zu zeigen sein wird, stehen sich kontroverse Positionen gegenüber, die im Kern auf Grundsatzentscheidungen über die Zukunft des Sozialstaates hinauslaufen. Zugleich ist zu erwarten, dass die Regelungen zur Hochwertung von Entgeltpunkten und zur Einführung von Freibeträgen bei der Grundsicherung und beim Wohngeld Anlass geben zu einer neuen Bestimmung der Sicherungsziele und des Leistungsniveaus der Rentenversicherung.

Wenig kontrovers - zumindest auf den ersten Blick - scheint die Zielbestimmung zu sein, dass Alterssicherung mehr ist und mehr sein soll als Armutsvermeidung und es nicht ausreicht, wenn das Gesamteinkommen im Alter nur ein wenig über dem Grundsicherungsniveau liegt. Einer Altersrente kommt vielmehr die Funktion zu, einen Ersatz für das Erwerbseinkommen zu leisten: Nach Aufgabe der Berufstätigkeit soll ein Einkommensabsturz nach dem Altersübergang verhindert und der im Laufe des Erwerbslebens erarbeitete und erreichte Lebensstandard weitgehend beibehalten werden können. Es geht auch und gerade für die Mitte der Gesellschaft um Einkommenskontinuität im Lebensverlauf, die Möglichkeit einer verlässlichen Einkommensplanung und um die Absicherung auch des Risikos von Erwerbsminderung und der Hinterbliebenenversorgung.

dann, wenn bezogen auf das gesamte Einkommen im Haushalt Bedürftigkeit besteht. Zudem muss zuvor verwertbares Vermögen aufgelöst werden, der Freibetrag liegt bei 5.000 Euro pro Person.

$4 \mathrm{Zu}$ einer ersten Modellrechnung vgl. Steffen 2020. 
Die große Gruppe von Beschäftigten im mittleren und gehobenen Einkommenssegment steht zwar auch in Zukunft nicht vor der Gefahr, im Alter unmittelbar in Armut zu geraten und auf die Grundsicherung angewiesen zu sein (vgl. Bäcker 2016a, S. 63 ff.). Aber die Sorge ist, infolge einer unzureichenden Alterssicherung den gewohnten Lebensstandard erheblich einschränken zu müssen ${ }^{5}$, d.h. vor allem die steigenden Mieten und die hohen Kosten einer pflegerischen Versorgung nicht mehr verkraften zu können.

Diese Aufgabe der Lebensstandardsicherung stellt sich nicht nur zum Zeitpunkt des Übergangs vom Arbeitsleben in den Rentenbezug, sondern im Verlauf der gesamten Altersphase, die bis zu drei Jahrzehnte dauern kann. Entscheidend ist also neben der Einkommenshöhe im Rentenzugang auch die laufende Anpassung der Alterseinkommen, um deren preisbedingte Wertminderung zu vermeiden und die Teilhabe an der allgemeinen Einkommens- und Wohlstandsentwicklung zu ermöglichen. Eine solche Anpassung ist das Kennzeichen der mit der Rentenreform von 1957 eingeführten lohnbezogenen Rentendynamik. ${ }^{6}$

\section{Rentenversicherung, Lebensstandardsicherung und Rentenniveau}

Allerdings erweist es sich alles andere als einfach, das Ziel der Lebensstandardsicherung zu konkretisieren. Zwar geht es allgemein gesehen um eine als „ausreichend“ angesehene Relation zwischen Erwerbseinkommen und Renten. Aber entscheidend für die gesetzlichen Renten ist, dass sie den gesamten Lebenslauf der Versicherten abbilden, dass ihre individuelle Höhe von der Dauer der versicherungspflichtigen Beschäftigung und von dem in dieser Zeit erzielten Einkommen abhängt. Es gilt das Prinzip der Teilhabeäquivalenz: Die in jedem Versicherungsjahr erworbenen und bei der Rentenberechnung schließlich addierten Entgeltpunkte widerspiegeln die in-

5 Die gerade im mittleren Einkommensbereich verbreitete Erwerbstätigkeit neben einem Bezug der Regelaltersrente („Arbeit trotz Rente“) ist nach den vorliegenden Forschungsbefunden nicht zuletzt ein Indiz für die Notwendigkeit, eine stark vom Arbeitseinkommen und bisherigen Lebenszuschnitt abweichende Altersrente aufzustocken, um sich z.B. einen Urlaub noch leisten zu können (vgl. Schmitz 2018, S. 99 ff.; Bäcker/Schmitz 2017, S. 229 ff.).

6 Selbst bei einer niedrigen Inflationsrate führt eine fehlende Dynamisierung zu einem erheblichen Kaufkraftverlust. Ein Beispiel: Eine Rente in konstanter Höhe von 1.000 Euro hat bei zwei Prozent Inflation pro Jahr nach 10 Jahren nur noch einen Wert von rund 820 Euro und nach 20 Jahren von rund 673 Euro. 
dividuelle Höhe des verbeitragten Einkommens im Verhältnis zum jeweiligen Durchschnittseinkommen. Wurde in einem Jahr genau im Durchschnitt verdient, so errechnet sich ein Entgeltpunkt in der Höhe von 1,0. Lag der Lohn unterhalb des Durchschnitts, z.B. bei $80 \%$, hat der Entgeltpunkt die Höhe von 0,8. In die Gesamtberechnung gehen damit sowohl niedrige Einkommenspositionen am Beginn des Berufslebens wie auch die in der Regel höhere Einkommenspositionen am Ende des Berufslebens ein. Abgesichert wird also die relative Einkommensposition im gesamten Versicherungsverlauf und nicht, wie bei der Beamtenversorgung, das Einkommen vor dem Berufsaustritt. Die auf das letzte Einkommen bezogene „Lohnersatzrate" kann deshalb sehr unterschiedlich ausfallen. Wenn beispielsweise das Einkommen vor dem Renteneintritt recht hoch ist und die persönliche lebensdurchschnittliche Einkommensposition übersteigt, dann fällt die Lohnersatzrate geringer aus. Bei einem Absinken der relativen Einkommensposition am Ende des Berufslebens tritt das Gegenteil ein. Hinzu kommt die strenge Verknüpfung zwischen Rentenanwartschaften und Versicherungsdauer. Wer nur wenige Jahre eingezahlt hat, kann keine Rente erwarten, die auch nur ansatzweise an das letzte Einkommen anschließt.

Das Sicherungsziel der Rentenversicherung bewegt sich in diesem Rahmen von individueller Rentenberechnung und allgemeiner Rentenanpassung (siehe weiter unten) und lässt sich durch die Maßgröße „Rentenniveau" beziffern. Seit 1992 (Rentenreform 1992) gelten 45 Versicherungsjahre als Norm für ein „erfülltes“ Arbeitsleben. Und der Zielwert des Rentenniveaus wurde damals bei rund $70 \%$ eines vergleichbaren Nettoarbeitnehmereinkommens angesetzt. Das heißt: Eine modellhaft ermittelte sogenannte Standardrente mit 45 Entgeltpunkten (im Verlauf von 45 Jahren wurde also durchschnittlich verdient) sollte bei $70 \%$ des aktuellen durchschnittlichen Nettoeinkommens der Arbeitnehmer liegen.

Bei dieser Berechnung von Höhe und Entwicklung des Rentenniveaus werden Nettogrößen (Nettorenten und Nettolöhne) miteinander verglichen, da es um die tatsächlich verfügbaren Einkommen geht. Allerdings können seit 2005 bei diesem Vergleich die Steuerabzüge nicht mehr berücksichtigt werden. Denn die seitdem geltende, schrittweise steigende Besteuerung der Renten variiert nach dem Jahr des Rentenzugangs: Je später der Rentenbeginn, desto höher fällt der Anteil der Rente aus, der der Besteuerung unterliegt. Im Ergebnis kann also von keiner für alle Rentner gleichen Steuerbelastung ausgegangen werden, so dass die Nettogrößen vor Steuern, aber nach Abzug von Sozialversicherungsbeiträgen einander 
gegenübergestellt werden müssen. In der Fachterminologie ist vom „Nettorentenniveau vor Steuern“ bzw. „Sicherungsniveau vor Steuern“ die Rede.

Der Zielwert von $70 \%$ lässt erkennen, dass das Sicherungsniveau seit Einführung der lohndynamischen Rente also niemals bei 100 Prozent lag; vielmehr galt eine Versorgungslücke von etwa $30 \%$ als akzeptabel, die entweder verkraftet werden muss oder durch ergänzende private und betriebliche Vorsorgeleistungen auszugleichen ist. So lag das Nettorentenniveau im Schnitt der 1980er und 1990er Jahre bei knapp 70 Prozent (mit einer Schwankungsbreite zwischen $72 \%$ und 66,9\%), was einem Nettoniveau vor Steuern von etwa 52 Prozent entspricht (Schäfer/Schmitz 2018, S. $21 \mathrm{ff}$.).

Da die Höhe des Rentenniveaus auf einer Modellrechnung beruht, lassen sich die Annahmen variieren:

- Werden - in Reaktion auf die Heraufsetzung der Altersgrenzen - bei den Versicherungszeiten mehr als 45 Jahre unterstellt, z.B. 47 Jahre, so fällt das Rentenniveau rechnerisch höher aus. Das ändert aber nichts am Entwicklungsverlauf des Niveaus (siehe unten) und an der Höhe irgendeiner Rente.

- Statt des Vergleichs von Durchschnittswerten (Standardrente und durchschnittliches Arbeitnehmereinkommen) können auch andere Konstellationen modelliert werden, indem z.B. eine Rente mit einer Einkommensposition von $70 \%$ (45 Versicherungsjahre x 0,7 EP $=31,5$ EP) einem Arbeitnehmerverdienst von $70 \%$ des Durchschnitts gegenübergestellt wird. Das so berechnete Niveau unterscheidet sich nicht von dem oben genannten Bezug auf die Standardrente.

\section{Anhaltender Rückgang des Rentenniveaus}

Die Entwicklung des Rentenniveaus im Zeitverlauf hängt im Wesentlichen von der jährlichen Rentenanpassung ab. Die absolute Höhe der individuellen Renten errechnet sich durch die Multiplikation der Summe der persönlichen Entgeltpunkte mit dem aktuellen Rentenwert. Der aktuelle Rentenwert ist eine Euro-Größe, er wird jährlich (zum 01.07.) in Anpassung an die Entwicklung der Arbeitsentgelte neu festgestellt. Bleibt die Anhebung des aktuellen Rentenwerts hinter der Lohnentwicklung zurück, so sinkt das Rentenniveau. Dies bedeutet nicht, dass die Renten in ihren 
absoluten Beträgen sinken (dies ist per Gesetz ausgeschlossen). Sie können durchaus weiter steigen - allerdings verlieren sie in der Relation zu den durchschnittlichen Löhnen an Wert.

Bei der Maßgröße des Rentenniveaus handelt es sich insofern um einen statistischen Indikator, der vor allem dazu dient, den Entwicklungsverlauf von Löhnen und Renten miteinander vergleichen zu können. Der Position von Rürup, der das Rentenniveau als „leere Monstranz“ bezeichnet (Rürup 2019), muss deshalb widersprochen werden; das Rentenniveau ist eine unverzichtbare „Kontrollinstanz“ (Schäfer/Schmitz 2018, S. 23), um überprüfen zu können, ob die Rentnergeneration (Zugangs- wie Bestandsrentner) am allgemeinen Einkommens- und Wohlstandszuwachs beteiligt wird oder nicht. Dieser Teilhabeanspruch hat nicht nur Bedeutung für die aktuelle Rentnergeneration, sondern auch für die nachrückenden Kohorten, die jetzt Beitragszahler sind und sich darauf verlassen müssen, im Alter ein ausreichendes Leistungsniveau zu erhalten.

Das Absinken des Rentenniveaus prägt die Situation der Rentenversicherung seit der Jahrtausendwende. Der Rückgang hat dabei schon vor 2000 eingesetzt, u.a. wirken sich hier die mehrfachen Anpassungsreduktionen aus. Weitreichende Folgen haben aber vor allem die Modifikationen der Rentenanpassungsformel, die im Zuge der Einführung der RiesterRente im Jahr 2001 und durch das RV-Nachhaltigkeitsgesetz von 2004 erfolgt sind:

- Veränderungen der Abgabenbelastung, die nicht die Alterssicherung betreffen (wie direkte Steuern, Beiträge zur Kranken-, Pflege- und Arbeitslosenversicherung), finden keine Berücksichtigung mehr.

- Anpassungsmindernd angerechnet werden Veränderungen des Beitragssatzes zur Rentenversicherung (in voller Höhe, also einschließlich des Arbeitgeberanteils).

- Zusätzlich angerechnet wird der private Beitrag für die staatlich geförderte zusätzliche Altersvorsorge. Unabhängig davon, ob er tatsächlich geleistet wird, wird ein Vorsorgebeitrag von $4 \%$ des Bruttoeinkommens unterstellt. Dieser anpassungsdämpfende Vorsorgefaktor wird als „Riester-Faktor" bezeichnet.

- Durch den Einbau eines „Nachhaltigkeitsfaktors“ in die Rentenanpassungsformel wird die Entwicklung des zahlenmäßigen Verhältnisses von Rentenbeziehern (sog. Äquivalenzrentner) und versicherungspflichtig Beschäftigten (Äquivalenzbeitragszahler) anpassungsmindernd wirksam. Sinkt - wie aus demografischen Gründen zu erwarten - der Rentnerquotient (Äquivalenzrentner zu Äquivalenzbeitragszah- 
lern), fallen die Rentenerhöhungen niedriger aus. Die jährliche Veränderung wird zu einem Viertel $(\boldsymbol{a}=0,25)$ bei der Rentenanpassung berücksichtigt. $^{7}$

Diese Veränderungen machen die Anpassungsformel hochkompliziert. Wichtig sind aber nicht die Details, sondern das Ergebnis: Die Rentenerhöhungen werden abgebremst und von den Einkommenszuwächsen der Aktiven mehr und mehr abkoppelt. Das Nettorentenniveau vor Steuern lag im Jahr 2018 noch bei 48,1\% und wird sich nach den Vorausberechnungen des Rentenversicherungsberichts 2019 bis 2033 auf 44,6\% verringern (Bundesregierung 2019). Gegenüber 2009 (Niveau von 52\%) entspricht dies einem Rückgang von 19,2\%. Mit anderen Worten: Ohne diese Eingriffe würde die Standardrente entsprechend höher ausfallen.

Dieser Abwärtstrend ist Ergebnis des 2001 eingeleiteten sog. „Paradigmenwechsels" in der Rentenpolitik. Als übergeordnetes Ziel gilt seitdem, die Beitragsbelastungen für die Versicherten und die Arbeitgeber zu begrenzen. Das Gesetz schreibt vor, den Beitragssatz - trotz der demografischen Belastungen - bis zum Jahr 2025 unter $20 \%$ und bis 2030 unter $22 \% \mathrm{zu}$ halten. Es dominiert der Grundsatz einer „einnahmeorientierten Ausgabenpolitik“. Hinsichtlich des Sicherungsziels gilt bis 2025 (geregelt im GRV-Leistungsverbesserungsgesetz von 2018) eine Haltelinie von $48 \%$ und bis 2030 von $43 \%$. Für die Zeit danach, in der der Nachhaltigkeitsfaktor stark anpassungsmindernd wirkt, gibt es keine Begrenzung mehr, die den Trend nach unten stoppen könnte. So prognostizierte das Bundesministerium für Arbeit und Soziales einen weiteren Rückgang bis auf 41,6\% im Jahr 2045 (BMAS 2016). Verschärfungen ergeben sich zusätzlich dadurch, dass die Renten dann voll besteuert werden.

Das Ziel der Lebensstandardsicherung setzt seitdem zwingend zusätzliche Leistungen aus der privaten und/oder betrieblichen Vorsorge voraus. Die Leistungen der gesetzlichen Rentenversicherung allein können und sollen das Ziel der Lebensstandardsicherung auf keinen Fall mehr errei-

7 Die gute Entwicklung auf dem Arbeitsmarkt insbesondere in den Jahren zwischen 2015 und 2018 hat zu einem deutlich gestiegenen Beschäftigungsniveau geführt. Die Zahl der Äquivalenzbeitragszahler hat sich zeitweise stärker entwickelt als die Zahl der Äquivalenzrentner, so dass das Rentenniveau stabilisiert worden ist. Wenn es bis 2024 keinen (konjunkturellen oder strukturellen) Beschäftigungseinbruch gibt, kann erwartet werden, dass die „Haltelinie“ von $48 \%$ auch ohne die im Gesetz vorgesehenen zusätzlichen Zuschüsse aus dem Bundeshaushalt nicht unterschritten wird. In den Jahren danach lässt sich aber die dann stark steigende Zahl der Äquivalenzrentner nicht mehr durch einen weiteren Beschäftigungszuwachs ausgleichen. 
chen. Leitbild ist die Lebensstandardsicherung aus mehreren Säulen. Neben die umlagefinanzierte Alterssicherung tritt - schrittweise und zu immer größeren Anteilen- die kapitalmarktabhängige Alterssicherung. Betriebliche und private Alterssicherung dienen also nicht mehr primär der Ergänzung der umlagefinanzierten Alterssicherung, sondern als Ersatz. Finanziert werden muss der Aufbau der privaten Vorsorge von den Versicherten bzw. Beschäftigten selbst, allerdings unterstützt durch staatliche Förderleistungen (steuerliche Freibeträge und Zulagen); als Zielgröße der Förderung gelten vier Prozent des Einkommens, eine paritätische Arbeitgeberbeteiligung wie bei der gesetzlichen Rente ist dabei nicht vorgesehen.

\section{Sinkendes Rentenniveau, Grundsicherungsbedarf und Grundrente}

Ein kontinuierlich sinkendes Rentenniveau bedeutet nun nicht nur, dass die Rentenanpassung hinter der Lohnentwicklung zurückbleibt. Zugleich erhöht sich auch die Wahrscheinlichkeit, dass trotz einer langjährigen Versicherungsdauer eine wachsende Zahl von Renten (Zahlbeträge) das Bedarfsniveau der Grundsicherung im Alter (einschließlich der Kosten der Unterkunft) nicht erreicht oder nur wenig darüber liegt. Um dies zu verdeutlichen, können Modellrechnungen vorgenommen werden (vgl. Abbildung 1). Geht man z.B. von einer lebensdurchschnittlichen Entgeltposition von $70 \%$ aus, dann waren im Jahr 2009 - bei einem damaligen NettoRentenniveau vor Steuern von $52 \%-37,4$ Versicherungsjahre erforderlich, um das Grundsicherungsniveau zu erreichen. Sinkt nun das Rentenniveau bis 2045 auf unter 41,6\% ab, dann reicht selbst diese lange Versicherungsdauer bei weitem nicht mehr aus. Erforderlich wären dann 47,7 Jahre.

Unterstellt wird bei dieser Modellrechnung, dass die Anpassungssätze von Grundsicherungsbedarf und aktuellem Rentenwert gleichgerichtet verlaufen. Das Gegenteil dürfte allerdings der Fall sein: Denn nach geltendem Recht fällt die jährliche Erhöhung der Regelbedarfe der Grundsicherung höher aus als die von der Lohnentwicklung abgekoppelte Anpassung der Renten. Die Fortschreibung der Regelbedarfe orientiert sich seit 2011 nicht mehr an der Erhöhung des aktuellen Rentenwerts, sondern an einem Mischindex, der auf der jährlichen Lohn- und Preisentwicklung im Verhältnis von $30 \% \mathrm{zu} 70 \%$ basiert. Erst in einem fünfjährigen Turnus, wenn die neuen Daten aus der Einkommensstatistik (Einkommens- und Verbrauchstichprobe, EVS) vorliegen, erfolgt dann eine Neubewertung der Regelbedarfe. Verschärfend kommt hinzu, dass die von der Grundsicherung übernommenen Kosten der Unterkunft infolge der Krise auf dem 
Wohnungsmarkt rapide - d.h. sehr viel stärker als der aktuelle Rentenwert - ansteigen. Schließlich ist damit zu rechnen, dass die Beitragssätze zur Kranken- wie auch zur Pflegeversicherung in den nächsten Jahren und Jahrzehnten steigen werden, so dass die Nettorenten vor Steuern (Zahlbeträge) geringer als unterstellt ausfallen.

Wenn eine gesetzliche Rente den Grundsicherungsbedarf unterschreitet, bedeutet dies trotz der zukünftigen Anrechnungsfreibeträge nicht, dass auch Anspruch auf Grundsicherungsleistungen besteht. Denn es ist zu berücksichtigen, dass die Einkommenslage stets im Kontext des Haushalts zu bewerten ist. Bei älteren Menschen ist hier in erster Linie an die verbreitete Konstellation der (Ehe-) Paar-Haushalte zu denken: Die Einkommen beider Partner fließen in den gemeinsamen Haushalt ein. Die niedrige Rente der Frau und die üblicherweise höhere Rente des Mannes bilden zusammen das verfügbare Gesamteinkommen. Und im Todesfall des Ehepartners/der Ehepartnerin ergänzen Hinterbliebenenrenten die eigene womöglich niedrige Rente.

Absehbar sind jedoch Legitimations- und Akzeptanzprobleme der Rentenversicherung, wenn nach jahrzehntelanger Beitragspflicht die individuelle Rente nicht oder nur kaum höher liegt als die vorleistungsunabhängige Grundsicherung im Alter und sich kein Unterschied mehr ergibt zu Personen, die keine oder keine entsprechend hohen Beiträge geleistet haben.

Dieses Problem einer Überschneidung von niedrigen Renten und Grundsicherungsniveau auch bei langjähriger Beitragszahlung wird durch die geplante Kombination von Grundrente und Freibeträgen bei der Grundsicherung und beim Wohngeld zweifelsohne gemildert. Die Entgeltpunkte werden angehoben und zugleich besteht infolge der Freibeträge ein erweiterter Anspruch auf Leistungen der Grundsicherung und des Wohngelds. Rentner*innen, die die Voraussetzung von 33 Versicherungsjahren erfüllen, werden dadurch gleich mehrfach bessergestellt. 
Abbildung 1: Überschneidung von Grundsicherungsbedarf und Rente bei sinkendem Rentenniveau nach Entgeltposition und Beitragsjahren, 2009-2045

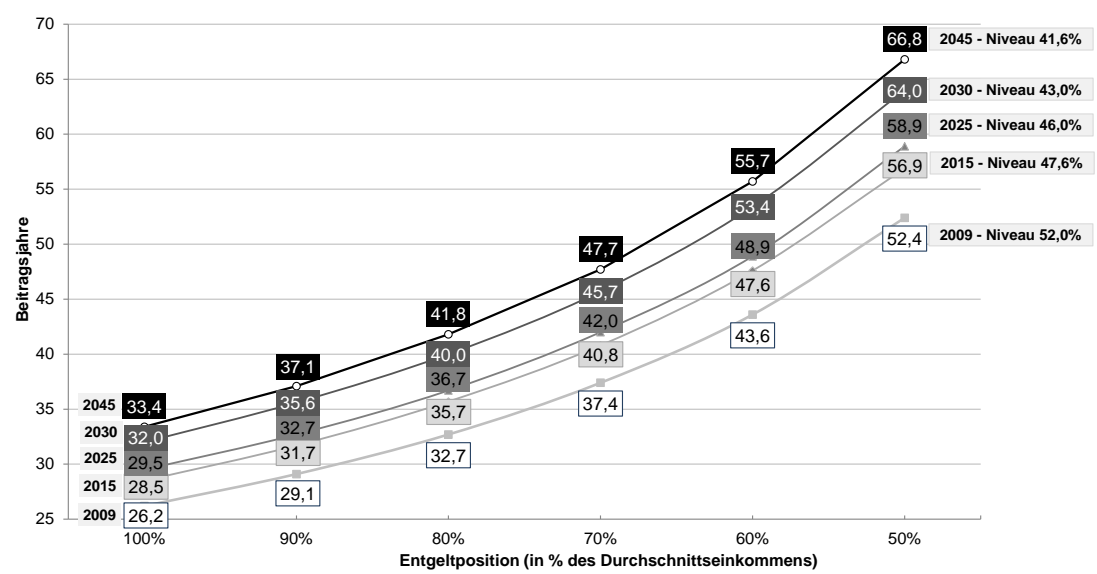

Quelle: eigene Berechnungen/Sozialpolitik-aktuell.de 2020. Annahmen (im Detail s. Sozialpolitik-aktuell.de 2020): Grundsicherung im Alter: Bedarf für Alleinstehende (Regelbedarf und bundesdurchschnittliche Kosten der Unterkunft) im Jahr 2015: 747 Euro; Rente: Nettorente vor Steuern, Beitragsabzüge (Krankenversicherung und Pflegeversicherung der Rentner) Werte für 2015; Niveau: 2009, 2015 (Deutsche Rentenversicherung), 2025 (Rentenversicherungsbericht 2015), 2030 (Niveausicherungsklausel), 2045 (Bundesregierung)

Es ist zu erwarten, dass dieses Ergebnis bei der Diskussion über den Bericht der Rentenkommission dazu verleiten wird, eine Absenkung des Rentenniveaus nach 2025 zu rechtfertigen, da sich die Folgewirkungen als weniger dramatisch oder gar unproblematisch herausstellen. Bei einer solchen Relativierung ist jedoch Widerspruch erforderlich:

- Die Freibeträge ändern nichts daran, dass Grundsicherungsansprüche den Zustand von „Bedürftigkeit“ im Haushaltskontext voraussetzen. Wenn das Einkommen eines Partners/einer Partnerin zu hoch ist, leistet die Grundsicherung nicht. Und auch ein vorhandenes Vermögen muss vorrangig eingesetzt werden; anrechnungsfrei bleibt lediglich ein Schonvermögen von 5.000 Euro.

- Durch die Absenkung des Rentenniveaus wird der Wert der Entgeltpunkte, also auch im Falle der Hochwertung, hinter der allgemeinen 
Einkommens- und insbesondere hinter der Entwicklung des Grundsicherungsniveaus zurückbleiben. Der Effekt der Hochwertung wird damit umso geringer, je stärker das Rentenniveau fällt.

- Die Aufwertung der Entgeltpunkte erreicht jene nicht, die mit ihrer lebensdurchschnittlichen Einkommensposition die Maximalschwelle von $0,8 \mathrm{EP}$ überschreiten.

Vor allem der letzte Punkt ist von grundsätzlicher Bedeutung: Wenn sich die öffentliche Alterssicherung auf die Absicherung am unteren Ende der Einkommensverteilung konzentriert (vgl. Klammer/Wagner 2020) und Renten und Grundsicherung schrittweise verschmelzen (vgl. Steffen 2019a; Schäfer 2014), besteht Gefahr, dass das Ziel der Lebensstandsicherung im Bereich mittlerer und auch höherer Einkommen aus den Augen verloren und immer stärker auf die private Altersvorsorge verschoben wird.

\section{Anhebung der Regelaltersgrenze und Rentenniveau}

Wie skizziert, lassen sich bei der Berechnung des Rentenniveaus nicht nur die zu vergleichenden Verdienstpositionen variieren, sondern auch die Zahl der Versicherungsjahre. Wenn statt der 45 Jahre 47 Jahre oder mehr unterstellt werden, erhöht sich allein rechnerisch gesehen das Rentenniveau, bei weniger als 45 Versicherungsjahren verringert es sich. In der aktuellen Debatte häufen sich nun die Aussagen, das Absinken des Rentenniveaus könne durch verlängerte Beitragszeiten gleichsam ungeschehen gemacht werden. Nach dieser Argumentation sinkt bei einer um zwei Jahre längeren Erwerbstätigkeit „das GRV-Verbersorgungsniveau bei Renteneintritt nicht von heute knapp $48 \%$ auf $44 \frac{1}{2} \%$ im Jahr 2029, sondern nur

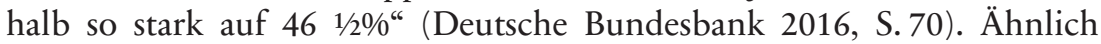
rechnet das Institut der deutschen Wirtschaft (2016): „Weist der Standardrentner im Jahr 2030 aufgrund der Rente mit 67 zwei Beitragsjahre mehr auf als heute, steigt das Rentenniveau vor Steuern von 44,6 auf 46,6 Prozent".

Allerdings handelt es sich hier um eine Fehlinterpretation des Rentenniveaus oder sogar um eine gezielte Irreführung. Denn wie auch immer verfahren und gerechnet wird, entscheidend ist, die Vergleichsgrößen konstant zu halten, um die Veränderung über die Zeit erkennen zu können. Wenn bei der Entwicklung im Zeitverlauf von einer unterschiedlichen Beitragsdauer ausgegangen wird (45 Jahre im Ausgangsjahr und 47 Jahre 
im Endjahr), wird die tatsächliche, nach unten gerichtete Entwicklung des Rentenniveaus zu verdeckt. Einen „dynamisierten Standardrentner“ (Sachverständigenrat 2016, S. 321) gibt es nicht.

Von diesen „Rechentricks“ zu unterscheiden ist die Frage, ob die Folgen des sinkenden Rentenniveaus durch eine Erhöhung der Beitragsjahre im Gefolge einer weiteren Anhebung der Regelaltersgrenze ausgeglichen werden können. Unsere Analyse zeigt, dass eine verlängerte Beschäftigungsund Beitragsdauer die Auswirkungen des sinkenden Rentenniveaus auf die zu erwartende Höhe der individuellen Rente in einem nur sehr begrenzten Ausmaß kompensieren kann. Die vorstehende Abbildung verdeutlicht, dass Beschäftigte im Bereich unterdurchschnittlicher Entgelte bereits aktuell und erst recht in der Zukunft keine Chance haben, eine Rente oberhalb des Grundsicherungsniveaus zu erreichen, da derart lange Versicherungsjahre schlicht unmöglich sind (Bäcker/Jansen/Schmitz, S. 120ff.). Der zu erwartende Rückgang des Rentenniveaus ist dabei so groß, dass selbst eine Verlängerung der Versicherungsdauer um 3 Jahre keine Entlastung bringt. Bei einem Rentenniveau von 41,6\% im Jahr 2045 sind bei einer Entgeltposition von $70 \%$ zusätzliche 6,9 Jahre gegenüber dem Stand von 2015 erforderlich, um eine Rente oberhalb des Grundsicherungsniveaus zu erreichen.

Eine weitere Heraufsetzung der Regelaltersgrenze löst also die Folgewirkungen des sinkenden Rentenniveaus keineswegs. Entscheidender noch aber ist, dass die als Problemlösung vorgestellte Verschiebung der Altersgrenze nach oben völlig an der Frage vorbei geht, ob die Arbeitnehmer/-innen hinsichtlich ihrer gesundheitlichen Konstitution und ihrer beruflichen Leistungsfähigkeit auch tatsächlich in der Lage sind, länger zu arbeiten. Und es ist ungewiss, wie sich der Arbeitsmarkt über 2030 hinaus entwickelt. Auf einen Automatismus, der sicherstellt, dass die Unternehmen immer Arbeitsplätze in ausreichender Zahl für die (weiterarbeitenden) Älteren bereitstellen, kann nicht gesetzt werden (vgl. dazu Bäcker 2018b, S. 997 ff.). Zu berücksichtigen sind die Unwägbarkeiten auf der Angebotsseite hinsichtlich der Größenordnung von Zuwanderung und Erwerbsbevölkerung, genauso wie auf der Nachfrageseite des Arbeitsmarktes hinsichtlich der Entwicklung der Zahl und Struktur der Arbeitsplätze. Von maßgebender Bedeutung für die Arbeitsnachfrage in den nächsten Jahren und Jahrzehnten ist die gesamtwirtschaftliche Entwicklung: Welches Wachstum ist zu erwarten, in welche Richtung weisen die Digitalisierung der Arbeitswelt und die Arbeitsproduktivität? 


\section{Private und betriebliche Altersvorsorge - hohe Erwartungen und ernüchternde Realität}

Die Frage ist, ob und inwieweit die Versorgungslücken durch den Ausbau der zweiten und dritten Säule der Alterssicherung gefüllt werden bzw. werden können (vgl. u.a. Schmähl 2012; S. 391 ff.; Steffen 2012, 413 ff). Grundlegende Voraussetzung dafür ist zunächst, dass alle Versicherten tatsächlich betrieblich und/oder privat ausreichend, frühzeitig und zugleich dauerhaft vorsorgen. Die empirischen Informationen dazu sind zwar äußerst spärlich, die wenigen vorliegenden empirischen Befunde lassen jedoch erkennen, dass dazu nur ein Teil der Beschäftigten entweder in der Lage oder bereit ist. So hat zwar die Zahl der Riester-Verträge (durch Zulagen und Steuervergünstigungen) in den Jahren bis 2015 bis auf 16,6 Mio. stark zugenommen. Seitdem gibt es - trotz steigender Bevölkerungs- und Beschäftigungszahlen - keinen Zuwachs mehr. Und 2019 ist sogar ein Rückgang erkennbar, der sich in den nächsten Jahren fortsetzen dürfte. Da eine Person mehrere Verträge abschließen kann, liegt die Zahl der Personen mit einem Vertrag noch niedriger. Die geförderte private Altersvorsorge steckt ersichtlich in einer Krise.

Allerdings kommt es nicht allein auf die Verbreitung der Riester-Verträge an. Viele Beschäftigte (insbesondere im öffentlichen Dienst und in den Großunternehmen der Privatwirtschaft) weisen eine zusätzliche Absicherung im Rahmen der betrieblichen Altersvorsorge auf. Zwar hat die Zahl der sozialversicherungspflichtigen Beschäftigten mit einer betrieblichen Altersvorsorge in den letzten Jahren leicht zugenommen, da aber gleichzeitig die Zahl der Beschäftigten noch stärker gestiegen ist, sinken die Anteilswerte (vgl. BMAS 2019, S. 14 f.) Insgesamt können trotz der finanziell aufwendigen Förderung allenfalls $60 \%$ der Beschäftigten (einschließlich Minijobber) irgendeine zusätzliche Alterssicherung aufweisen. Außen vor stehen vor allem jene, die sich im unteren Arbeitsmarkt- und Beschäftigungssegment verorten lassen. Auch die mit dem Betriebsrentenstärkungsgesetz von 2017 verbundenen Erwartungen einer deutlichen Ausdehnung von Betriebsrenten im Rahmen des sog. Sozialpartnermodells (mit reinen Beitragszusagen) haben sich bislang nicht erfüllt.

Selbst wenn der Verbreitungsgrad bei der zusätzlichen Altersvorsorge höher läge, so fehlen doch verlässliche Informationen über die Höhe der zu erwartenden Rente. Die vorliegenden Daten über die Zahl von Verträgen beziehen sich nämlich nur auf einen Zeitpunkt (2. Quartal 2019) und lassen keinerlei Aussagen über die Leistungshöhe zu, die sich ja erst im Zeitverlauf der Vorsorge zeigen. Denn die Höhe einer kapitalfundierten Rente hängt grundlegend davon $a b$, wie hoch die Sparbeträge sind und 
wie lange eingezahlt wird. Nur eine langjährige, kontinuierliche Einzahlung - beginnend mit dem Berufseintritt und endend mit dem Berufsaustritt - garantiert eine hohe Rente. Die vorliegenden Daten geben darüber keine Auskunft. Unklar ist, ob es sich um langfristig bediente oder Neuverträge handelt, in welchem Lebensalter sich die Vertragsnehmer befinden, welche Höhe die Sparbeträge aufweisen ${ }^{8}$ und ob Verträge ausgelaufen oder gekündigt sind. Allein die Information, dass mittlerweile mehr als ein Fünftel (!) der Verträge „ruhend gestellt“ sind (vgl. BMAS 2020), also aktuell nicht mehr bedient werden, lässt erkennen, dass sich hier erhebliche Absicherungslücken auftun.

Welche konkrete Höhe eine kapitalgedeckte Rente zum Auszahlungsbeginn und während des gesamten Auszahlungszeitraums aufweisen wird, lässt sich nicht prognostizieren. Die Rendite einer kapitalfundierten Altersvorsorge steht und fällt mit der Entwicklung auf den Kapitalmärkten. Für eine einzelne Person kommt es dabei nicht auf irgendwelche Durchschnittsrenditen an, was zählt ist die schlussendliche Verzinsung, also der Vergleich zwischen den Einzahlungsbeginn und dem konkreten Auszahlungsbeginn (vgl. vor allem Fachinger 2018, S. 173 ff.). Angesichts der wohl dauerhaften und weltweiten Niedrigzinsphase werden die Renditen „sicherer“ Altersvorsorgeverträge in Zukunft ausgesprochen niedrig ausfallen. Die Reduktion der Garantiezinsen für Neuverträge deutet an, wohin die Reise geht. Und in Abzug gebracht werden müssen die hohen Vertriebs- und Akquisitionskosten. Sicherheit gewährt bei Riester-Produkten allein die Nominalwertgarantie: Mindestens alle geleisteten Beiträge einschließlich der Zulagen müssen bei Beginn der Auszahlungsphase zur Verfügung stehen.

Die Bundesregierung zeigt sich in ihren jährlichen Rentenversicherungsberichten von diesen systemischen Schwächen der kapitalbasierten Altersvorsorge unbeeindruckt. Ausdrücklich festhaltend an dem Ziel einer Lebensstandardsicherung mit einem Sicherungsniveau von $52 \%$ (vor Steuern) wird vorgerechnet, dass im Mehrsäulenmodell das Gesamtversorgungsniveau - basierend auf der Standardrente und einer Riester-Rente bis 2033 diesen Zielwert nur knapp unterschreitet bzw. zwischenzeitlich sogar übersteigt (2025: 53,2 \%; 2033: 51,7\%; vgl. Bundesregierung 2019, Übersicht B 8). Schaut man genauer hin, dann handelt es sich bei dieser

8 Der Förderstatistik der Zulagenstelle ist zu entnehmen, dass zum Auswertungsstichtag 15.05.2018 unter den 11 Mio. Geförderten nur rund $53 \%$ die volle Förderung erhalten und gut $20 \%$ weniger als die Hälfte des Vorsorgebeitrags von vier Prozent des Bruttoeinkommens leisten (vgl. Bundesministerium der Finanzen 2019). 
Modellrechnung lediglich um eine Schönrechnerei. Denn die Modellannahmen gaukeln eine irreale Welt vor: Seit 2002 wird flächendeckend und zeitlich lückenlos geriestert, seit 2008 mit einem Vorsorgeaufwand von vier Prozent des Durchschnittsentgelts (volle Sparleistung und Zulagen), und die Realverzinsung des Altersvorsorgekapitals liegt langfristig bei vier Prozent pro Jahr. Zu einem Erkenntnisgewinn führen diese Berechnungen eines Gesamtversorgungsniveaus deshalb nicht (vgl. Steffen 2019b; Schäfer 2015). Zudem bleibt unberücksichtigt, dass die geförderten Riester-Renten - anders als bei der Rentenversicherung - weder die Risiken von Erwerbsminderungen und Hinterbliebenenversorgung abdecken noch mit einer expliziten Anpassungsdynamik ausgestaltet sind. Das Gesamtversorgungsniveau im Jahr des Rentenzugangs lässt nicht erkennen, dass mit einem Rückgang im Laufe des Rentenbezugs gerechnet werden muss.

\section{Lebensstandardsicherung durch Obligatorium und Standardprodukt?}

Trotz aller Negativerfahrungen mit der Teilprivatisierung der Alterssicherung mehren sich in der aktuellen Debatte die Stimmen (nicht zuletzt von Mitgliedern der Rentenkommission der Bundesregierung), die den Paradigmenwechsel bekräftigen und an dem Konzept eines sinkenden Rentenniveaus und einer Lebensstandardsicherung mit Leistungen aus mehreren Säulen festhalten wollen. Um den Grundgedanken eines Ausgleichs des sinkenden Rentenniveaus zu „retten“, soll vom bisherigen Prinzip der Freiwilligkeit (gefördert durch finanzielle und steuerliche Anreize) abgewichen und eine Verallgemeinerung der kapitalmarktabhängigen Altersvorsorge erreicht werden, bei der dann auch die Grenzen zwischen privater und betrieblicher Vorsorge verschwimmen. Die Modelle wie auch die entsprechenden Bezeichnungen sind vielfältig: Bekannt sind vor allem die Konzepte „Vorsorgekonto“ (vgl. Stellpflug et al. 2009; zur Einschätzung: Thiede/Rieckhoff 2019, S. 193 ff.), „Deutschland Rente“ (Hessisches Ministerium der Finanzen 2017; zur Einschätzung Bäcker 2016b, S. 143 ff.) und „Extra-Rente“ (VZBV 2019). Zu unterscheiden ist hinsichtlich der Ausweitung zwischen einer Absicherungspflicht (Obligatorium) für alle sozialversicherungspflichtigen Beschäftigten oder einer (eingeschränkten) Freiwilligkeit, bei der die Beschäftigten zwar durch den Arbeitgeber automatisch angemeldet werden, aber die Möglichkeit des Widerspruchs haben (opting-out).

Verknüpft ist diese Orientierung hin zu einer möglichst flächendeckenden Verbreitung der zusätzlichen Altersvorsorge mit der Vorstellung, ein staatlich organisiertes Standard- oder Basisprodukt (oder auch mehrere, 
nach Risikoklassen differenzierte) Produkte bereit zu stellen, in das die Beschäftigten automatisch einbezogen werden und einzahlen - gegebenenfalls mit der Möglichkeit des opting-out. Die Regelungen in einzelnen skandinavischen Ländern und auch in Großbritannien dienen hier als Vorbild. Statt der unübersichtlichen, teuren und renditeschwachen Vorsorgeprodukte, die von Banken und Versicherungen im Rahmen der RiesterFörderung angeboten werden, soll dieses Standardprodukt einfach, transparent und kostengünstig sein. Die Verwaltung der individuellen Vorsorgekonten wie auch die Kapitalanlage soll durch eine staatliche bzw. öffentliche Institution (z.B. Rentenversicherung oder Bundesbank) erfolgen. Durch die Ermöglichung, auch weltweit in Aktien und Fonds zu investieren und auf Garantien hinsichtlich Auszahlungshöhe und Kapitalwerterhalt weitgehend zu verzichten, sollen die Renditen auch im Niedrigzinsumfeld ausreichend hoch ausfallen.

Ein solch umfassender Einstieg der Alterssicherung in die Welt der Kapital- und Finanzmärkte wirft jedoch mehr Fragen und Probleme auf als Antworten und Lösungen zu bieten:

- Selbst ein alle Erwerbstätigen einbeziehendes Obligatorium würde nicht zu einer flächendeckenden Absicherung führen. Denn Personen, die wegen Krankheit, Behinderung, Kindererziehung, Pflege oder Arbeitslosigkeit zwischenzeitlich oder gar längerfristig nicht berufstätig sind, bleiben im Alter unversorgt oder unterversorgt.

- Einen Solidarausgleich kennt die private Vorsorge nicht. Es herrscht das reine Äquivalenzprinzip von Beitrag und Leistung. Beitragszahlungen des Bundes oder anderer Versicherungsträger für Zeiten von u.a. Krankengeldbezug, Elternschaft, Arbeitslosigkeit und Pflege gibt es ebenso wenig wie Ausgleichselemente bei der Rentenberechnung für Zeiten geringer Verdienste (Höherwertung, Zurechnungszeiten, Anrechnungszeiten). $\mathrm{Zu}$ einer Umverteilung führt lediglich die staatliche Förderung durch Zulagen bzw. Steuervergünstigungen (Sonderausgabenabzug). Durch die Abhängigkeit der Höhe der Zulagen vom Einkommen und vor allem nach der Kinderzahl zeigt sich eine soziale Komponente. Auf der anderen Seite führt die steuerliche Förderung, die bei Beziehern hoher Einkommen günstiger ist als die Zulagenförderung, zu starken Mitnahmeeffekten, da die private Altersvorsorge ohnehin erfolgt.

- Schwierig ist es, neben dem Alter auch die Risiken Erwerbsminderung und Tod (Hinterbliebenenrenten) abzudecken. Dies ist zwar durchaus möglich, wäre aber wohl mit deutlichen Abstrichen beim Leistungsni- 
veau der Altersrenten verbunden. Und ungelöst ist, wie eine ausreichend hohe private oder betriebliche Rente bei Erwerbsminderung gewährleistet werden soll, wenn die Erwerbsminderung bereits in jüngeren Jahren eintritt und keine Möglichkeiten bestanden hat, ausreichend lange vorzusorgen.

- Wenn die Betriebe die individuellen Sparleistungen in einem mehr oder minder obligatorischen System an die Kontenverwaltungsstelle weitergeben, so ist dies sicher ein einfaches und betriebs- wie branchenübergreifendes Verfahren. Aber es fragt sich, welcher Unterschied dann überhaupt noch zur Finanzierung der Rentenversicherung durch Beiträge besteht. In beiden Fällen mindern sich die Einkommen - allerdings werden bei der zusätzlichen Vorsorge nur die Beschäftigten belastet, weil nicht zu erwarten ist, dass sich die Arbeitgeber paritätisch beteiligen.

- Angesichts der aktuellen und auch zukünftigen Probleme auf den internationalen Kapitalmärkten ist es gewagt, von hohen Anlagerenditen auszugehen. Wenn tatsächlich alle Beschäftigten in Deutschland (und auch in vielen anderen Staaten) zusätzlich vorsorgen, dauerhaft mit etwa vier Prozent ihres Einkommens, errechnen sich riesige Anlagevolumina, die auf den Märkten untergebracht werden müssen. Der Verweis auf Anlagen in Aktien, verbunden mit einem weitgehenden Verzicht auf Garantien, ist zwar mit Chancen, aber auch mit unwägbaren Risiken verbunden. Renditen lassen sich eben nicht garantieren; und sie unterscheiden sich je nach Jahr des Beginns der Einzahlung und der dann folgenden Auszahlung. Deshalb ist es bei kapitalgedeckten und damit kapitalmarktabhängigen Altersvorsorgeleistungen systemisch überhaupt nicht möglich, ein definiertes Leistungsziel „Lebensstandardsicherung“ (mit einer Maßgröße) vorzugeben. Erst recht ist nicht abschätzbar, ob und inwieweit die kapitalmarktabhängigen Renten im Laufe der Ruhestandsphase an die allgemeine Einkommens- und Preisentwicklung angepasst werden (vgl. Fachinger et al. 2015, S. 303 ff.).

\section{Langfristige Stabilisierung des Rentenniveaus als vorrangige Aufgabe}

Die Analyse der Schwachstellen der privaten und betrieblichen Altersvorsorge lässt erkennen, wie problematisch es ist, die kapitalmarktabhängige Alterssicherung als tragende Säule zu verstehen und zu etablieren. Betriebsrenten, private Leibrenten und andere Vorsorgeprodukte können die Leistungen der umlagefinanzierten Rentenversicherung ergänzen aber eben nicht ersetzen. Dies ist der entscheidende Punkt, der in der aktuellen 
Debatte über die Zukunft des „Drei-Säulen-Modells“ zu beachten ist. Reformvorschläge, die die kapitalfundierte Altersvorsorge ausweiten und revitalisieren wollen, zugleich aber den kontinuierlichen Rückgangs des Rentenniveaus akzeptieren, sind Scheinlösungen, die die Risiken von fehlender Lebensstandardsicherung verstärken statt zu mindern. Sie gleichen einer Therapie, die die Wirksamkeit einer falschen Medizin durch eine höhere Dosis verbessern will.

Wenn Konsens darüber besteht, dass es Aufgabe des Sozialstaates ist, im Alter den Lebensstandard auf einem verlässlichen Niveau zu sichern, führt an einer Stärkung der gesetzlichen Rentenversicherung kein Weg vorbei. Es war deshalb ein wichtiges Signal, dass die große Koalition eine Haltelinie beim Rentenniveau - allerdings nur bis zum Jahr 2025 - fixiert hat. Bis dahin soll das Niveau bei 48 Prozent gehalten werden. Die im RV-Leistungsverbesserungsgesetz von 2018 formulierte Begründung, die die Bundesregierung für diese Niveaustabilisierung nennt, gilt zweifelsohne auch für die Jahre danach: „Ein angemessenes und stabiles Sicherungsniveau vor Steuern ist wichtig für die Akzeptanz der gesetzlichen Rentenversicherung. Es muss generationenübergreifend vertrauensbildende Zusagen geben“" (Bundesregierung 2018, S.1). Weitergehende Forderungen in Richtung einer Wiederherstellung des vor der Jahrtausendwende bestehenden Niveaus liegen zwar nahe, sind aber allein aus finanziellen Gründen wohl unrealistisch, da über Jahre hinweg die Rentenanpassung stärker als die Lohnentwicklung ausfallen müsste - verbunden mit stark steigenden Beitragssätzen. Viel wichtiger ist die Aufgabe, eine Stabilisierung auch nach 2025 sicherzustellen, in einer Zeit, in der die geburtenstarken Jahrgänge das Rentenalter erreichen und die Ausgaben entsprechend stark nach oben weisen.

Diese Orientierung schließt nicht aus, die Bedingungen einer ergänzenden, aber eben nicht ersetzenden privaten und betrieblichen Vorsorge zu verbessern (vgl. im Detail Fassauer/Petersen/Rieckhoff 2018, S. $119 \mathrm{ff}$ ). Insbesondere die Entwicklung eines übersichtlichen, preisgünstigen und öffentlich verwalteten Vorsorgeproduktes würde die Position Verbraucher stärken - stößt verständlicherweise auf keine Gegenliebe des Finanz- und Versicherungssektors, da sich daran ja kaum verdienen lässt (vgl. Bäcker 2016b, S. 144). Verwegen wäre es allerdings, sich hiervon „Renditewunder" zu versprechen. Eine Abkopplung von der allgemeinen Entwicklung des Kapitalmarkts ist nicht möglich.

Die Finanzierungsbedingungen infolge der Alterung der Gesellschaft erweisen sich als nicht einfach. Der Beitragssatz zur gesetzlichen Rentenversicherung liegt aktuell mit $18,6 \%$ so niedrig wie seit über zwanzig Jahren nicht mehr (1999: 20,3\%). Nach der 2016 vom BMAS vorgelegten Berech- 
nung würde er bei einer Fortgeltung der Haltelinie von rund $48 \%$ bis auf etwa 26,9\% steigen. Von Dramatik sollte aber nicht gesprochen werden. Denn die Entwicklung vollzieht sich über einen Zeitraum von mehreren Dekaden, sprunghafte Beitragssatzanstiege muss es deshalb nicht geben. Wichtig für die Zahlungsbereitschaft und Zahlungsfähigkeit im generativen Übertragungsprozess ist dabei, welche Höhe die individuellen Einkommen haben, die in Zukunft erwirtschaftet werden. Die Einkommensentwicklung entscheidet, ob es gelingt, steigende Beitragsbelastungen auch ohne Realeinkommensverluste zu verkraften. $\mathrm{Zu}$ berücksichtigen sind also die gesamtwirtschaftlichen Trends, nämlich die Zuwachsraten von Beschäftigung, Sozialprodukt, Produktivität und Arbeitseinkommen. Eine einfache Rechnung kann den Zusammenhang verdeutlichen: Wenn ein jahresdurchschnittlicher Anstieg der durchschnittlichen pro-Kopf Arbeitnehmereinkommen (brutto) von real 1,5\% unterstellt wird, errechnet sich in 40 Jahren eine Erhöhung um gut 80 \%. Bei einer Erhöhung von $1 \%$ im Jahresdurchschnitt sind es immer noch rund $50 \%$. Der Verteilungsspielraum wächst und trotz steigender Beitragssätze kommt es zu Reallohnsteigerungen. Unter diesen Bedingungen, also aus einem steigenden Wohlstand heraus, müssen und können die demografischen Veränderungen bewältigt werden.

Vor allem aber ist nach dem Sinn von vorgeschlagenen Alternativen zu fragen (vgl. Bäcker 2018a, S. 172 ff.). Soll der Lebensstandard in der Ruhestandsphase gesichert werden, dann müssen bei einem sinkenden Rentenniveau anderweitige Maßnahmen ergriffen werden, um diese Ziele zu erreichen. „Kostenlos“ ist dies nicht zu haben. Auch der geforderte Ausbau der kapitalfundierten Systeme ändert daran nichts. So sind die Sparbeträge bei der Riester-Rente, die Beiträge zu einem Standardprodukt wie auch die arbeitnehmerfinanzierte betriebliche Altersversorgung im Rahmen der Entgeltumwandlung mit Einkommensabzügen von mindestens vier Prozent verbunden, die allerdings nur die Arbeitnehmer und nicht die Arbeitgeber belasten. Warum sind höhere Beitragssätze in der Rentenversicherung „unbezahlbar“ (Axel Börsch-Supan in der Süddeutschen Zeitung vom 23.04.2018; Börsch-Supan/Rausch 2018), die Einkommensabzüge im Rahmen einer (weitgehend) verpflichtenden privaten Vorsorge aber nicht? Bei der Suche nach einer Antwort auf diese Frage ist es wohl nicht falsch, auch auf die Geschäftsinteressen des Finanzsektors zu verweisen.

Höhere Beitragssätze zur Rentenversicherung dürfen deshalb nicht zum Tabu erklärt werden. Es bleibt die Einsicht, dass sich das realwirtschaftliche Problem des demografischen Umbruchs, dass mehr Ältere müssen durch weniger Jüngere versorgt, werden müssen, nicht durch die Wahl einer anderen Finanzierungsform umgehen lässt. Es ist ein Gebot der poli- 
tischen Ehrlichkeit, dies zu verdeutlichen, statt die höhere Belastung der Arbeitnehmer in den Modellen der privaten Vorsorge gleichsam zu verstecken.

\section{Literatur}

Bäcker, G. (2016a): Altersarmut, Lebensstandardsicherung und Rentenniveau, in: Naegele, G./Obermann, E./Kuhlmann, A. (Hrsg.), Teilhabe im Alter gestalten, Wiesbaden, S. 63-82

Bäcker, G. (2016b): Runderneuerung der Riester-Rente: Mehr von der falschen Medizin?, in: Soziale Sicherheit 65 (4), S. 143-147

Bäcker, G. (2018a): Ist ein stabilisiertes Rentenniveau unbezahlbar?, in: Soziale Sicherheit 67 (5), S. 172-183

Bäcker, G. (2018b): Wann und Wie in den Ruhestand? Altersübergänge im Umbruch. In: Sozialer Fortschritt 67 (11-12), S. 997-1016

Bäcker, G./Jansen, A./Schmitz, J. (2017): Rente erst ab 70? Probleme und Perspektiven des Altersübergangs, IAQ-Forschung 02/2017, Duisburg

Bäcker, G./Schmitz, J. (2017): Über eine Millionen Menschen ab 65 sind erwerbstätig. Erwerbstätigkeit im Ruhestand, in: Soziale Sicherheit 66 (6), S. 229-236

BMAS (Bundesministerium für Arbeit und Soziales, 2016): Das Gesamtkonzept der Alterssicherung. Das Konzept im Detail, Berlin

BMAS (2019): Trägerbefragung zur Verbreitung der betrieblichen Altersversorgung - Endbericht, Forschungsbericht 523, Berlin

BMAS (2020): Statistik zur privaten Altersvorsorge, https://www.bmas.de/DE/The men/Rente/Zusaetzliche-Altersvorsorge/statistik-zusaetzliche-altersvorsorge.html (abgerufen am 13.02.2020)

Börsch-Supan, A./Rausch, J. (2018): Die Kosten der doppelten Haltelinie, MEA Discussion Paper 03-2018, München

Bundesministerium der Finanzen (2019): Statistische Auswertungen zur RiesterFörderung, Auswertungsstichtag 15. Mai 2018 - Beitragsjahre 2014 bis 2017, https://www.bundesfinanzministerium.de/Content/DE/Standardartikel/Themen /Steuern/Weitere_Steuerthemen/Altersvorsorge/2018-11-14-Statistische-Auswert ungen-Riester-Foerderung-bis-2017.html (abgerufen am 13.02.2020)

Bundesregierung (2018): Entwurf eines Gesetzes über Leistungsverbesserungen und Stabilisierung in der gesetzlichen Rentenversicherung, Bundestags-Drucksache $19 / 4668$

Bundesregierung (2019): Rentenversicherungsbericht 2019, Berlin

Deutsche Bundesbank (2016): Exkurs: Zur längerfristigen Entwicklung der Alterssicherung, in: Monatsbericht August, S. 69-77

Fachinger, U. (2018): Die Dynamisierung von Alterseinkommen. Chancen und Herausforderungen im Mehrschichtsystem, in: Sozialer Fortschritt 67 (3), S. $173-195$ 
Fachinger, U./Künemund, H./Schulz, M.F./Unger, K. (2015): Kapitalgedeckte Altersversorgung: Ihr Beitrag zur Lebensstandardsicherung, in: Fachinger, U./ Schmähl, W. (Hrsg.): Absicherung im Alter. Diskurse und Perspektiven, Münster, S. 303-349

Fassauer, St./Petersen, I./Rieckhoff, Ch. (2018): Bedarf die staatliche Förderung der Riester-Rente der Veränderung? Ausgewählte Reformüberlegungen in der Diskussion, in: Deutsche Rentenversicherung 02/208, S. 119-143

Hessisches Ministerium der Finanzen u.a. (2017): Deutschland-Rente, Konzeptpapier, Wiesbaden

Institut der deutschen Wirtschaft (2016): Rente realistisch gerechnet, https://www.i wd.de/artikel/rente-realistisch-gerechnet-311307/ (abgerufen am 13.02.2020)

Klammer, U./Wagner, G. G. (2020): Grundrentenplan der großen Koalition. Welche Risiken soll die gesetzliche Rentenversicherung versichern? In: Wirtschaftsdienst 100 (1), S. 29-34

Knabe, A./Weimann, J. (2018): Die Deutschlandrente - Wirksamkeit und Legitimität eines Nudges., in: Vierteljahreshefte zur Wirtschaftsforschung 02/2018, S. 33-46

Rürup, B. (2019): Das Rentenniveau: Eine leere Monstranz, in: Handelsblatt Research Institute, https:/www.handelsblatt.com/politik/konjunktur/research-insti tute/der-chefoekonom-das-rentenniveau-eine-leere-monstranz/24856930.html?tic ket=ST-1544181-ntzFGj59N4JMbn10hf3U-ap6, 02.08.2019 (abgerufen am 13.02.2020)

Sachverständigenrat zur Begutachtung der gesamtwirtschaftlichen Entwicklung (2016): Zeit für Reformen. Jahresgutachten 2016/17, Wiesbaden

Schäfer, I. (2014): Die Vollendung eines Paradigmenwechsels, in Vierteljahreshefte zur Wirtschaftsforschung 02/2014, S. 21-32

Schäfer, I. (2015): Die Illusion von der Lebensstandardsicherung. Eine Analyse der Leistungsfähigkeit des „Drei-Säulen-Modells“, Schriftenreihe der Arbeitnehmerkammer Bremen 01/2015, Bremen

Schmähl, W. (2012): Gründe für einen Abschied von der „neuen deutschen Alterssicherungspolitik" und Kernpunkte einer Alternative, in: Bispinck, R./Bosch, G./Hofemann, K./Naegele, G. (Hrsg.), Sozialpolitik und Sozialstaat, Wiesbaden, S. 391-412

Schmitz, J. (2018): Erwerbstätigkeit im Rentenalter: sozialpolitische Probleme und Implikationen, in: Scherger, S./Vogel, C. (Hrsg.): Arbeit im Alter, Zur Bedeutung bezahlter und unbezahlter Tätigkeiten in der Lebensphase Ruhestand, Wiesbaden, S. 99-123

Schmitz, J./Schäfer, I. (2018): Das Rentenniveau: Messverfahren, Einflussfaktoren und Fehlinterpretationen, in: Soziale Sicherheit 67 (1), S. 21-25

Sozialbeirat (2019): Gutachten des Sozialbeirats zum Rentenversicherungsbericht 2019, https://sozialbeirat.de/dokumente/ (abgerufen am 21.03.2020) 
Sozialpolitik-aktuell.de (2020): Überschneidung von Grundsicherungsbedarf und Rente bei sinkendem Rentenniveaunach Entgeltposition und Beitragsjahren, 2009-2045, http://www.sozialpolitik-aktuell.de/tl_files/sozialpolitik-aktuell/_Pol itikfelder/Alter-Rente/Datensammlung/PDF-Dateien/abbVIII54.pdf (abgerufen am 13.02.2020)

Steffen, J. (2012): Lebensstandardsicherung und Armutsfestigkeit im „Drei-SäulenModell“ der Alterssicherung, in: Bispinck, R./Bosch, G./Hofemann, K./Naegele, G. (Hrsg.), Sozialpolitik und Sozialstaat, Wiesbaden, S. 413-425

Steffen, J. (2019a): Grundsicherung und Altersrente - Ein Prozess systemischer Verschmelzung, www.portal-sozialpolitik.de/index.php?page=grundsicherung-alters rente-verschmelzung, Oktober 2019 (abgerufen am 13.02.2020)

Steffen, J. (2019b): Rentenversicherungsbericht 2019: Gesamtversorgungsniveau: Mehr Erklärungsbedarf als Erkenntnisgewinn, http:/www.portal-sozialpolitik.d e/index.php?page=rentenversicherungsbericht (abgerufen am 13.02.2020)

Steffen, J. (2020). Grundrente: Die drei Komponenten: Ein widersprüchliches Zusammenspiel, in: http://www.portal-sozialpolitik.de/index.php?page=grundrente -referentenentwurf (abgerufen am 13.02.2020)

Stellpflug, J./Strenberger-Frey, B./Tuchscherer, C. (2019): Das Vorsorgekonto - Basisprodukt für die private Altersvorsorge, WISO-Diskurs 01/2019, Bonn

Thiede, R./Rieckhoff, Ch. (2019): Das „Vorsorgekonto“- (zu) viele Fragen und Widersprüche, in: Deutsche Rentenversicherung 03/2019, S. 193-205

VZBV (Verbraucherzentrale Bundesverband) (2019): Die Extrarente: Freiwillig. Fair. Einfach. Mehr, Forderung des vzbv für ein Standardprodukt zur privaten Altersvorsorge, Berlin 